\title{
Roadshow
}

\section{Professionelle Aufklärung zum Schutz vor Zahnfleischerkrankungen}

Mehr als 70\% aller Erwachsenen ab 35 Jahren sind von einer Parodontitis betroffen. Parodontaler Attachment- und Knochenverlust hängt von allgemeingesundheitlichen und erworbenen Risikofaktoren sowie von der Mundhygienesituation ab. Daher ist eine frühzeitige und erfahrbare professionelle Aufklärung auch bereits vor dem 35. Lebensjahr sinnvoll und wichtig. Hier greift das Prinzip der meridol ${ }^{\circledR}$-Testwochen. Ziel dieser Testwochen ist es, flächendeckend besonders viele Bevölkerungsgruppen in ungewohntem Umfeld und unter professioneller Anleitung über die Risiken von Zahnfleischerkrankungen aufzuklären. Teil des Roadshow-Konzepts ist es, Präventionsmaßnahmen erlebbar zu machen und damit zur besseren Vorsorge beizutragen. Neben der Ermunterung zur regelmäßigen zahnärztlichen Kontrolle sowie einer sinnvollen, durchführbaren häuslichen Mundhy-

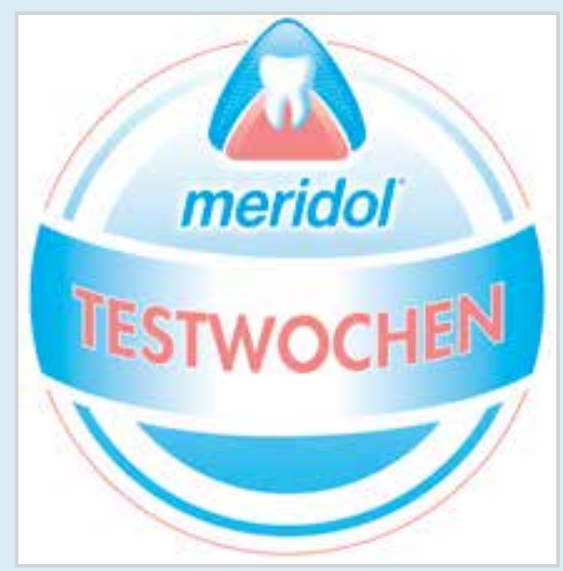

giene werden die Betroffenen zudem über die besonderen Wirkstoffe und Technologien der meridol-Produkte informiert. Die Roadshow ist vom 20. März bis zum 30. Mai unterwegs. Zahnärzte und zahnmedizinisches Fachpersonal informieren in diesem Zeitraum in 10 deutschen Städten „auf der Plaza“ in großen Einkaufszentren rund um das Thema Zahnpflege. Neben Tipps für die optimale Mundhygiene mit dem meridol-System wird den Besuchers des meridol-Testwochenstands empfohlen, regelmäßig ihren Zahnarzt zu besuchen und entsprechende Prophylaxeleistungen in Anspruch zu nehmen. Außerdem haben die Besucher die Möglichkeit, sich an einem Touchscreen selbst zu testen, indem sie Fragen zu ihrer Zahnfleischgesundheit und ihren Zahnputzgewohnheiten beantworten.

Die Termine und Orte der meridol-Roadshow 2015:

30.4.-02.5. Hamburg

08.5.-09.5. Frankfurt

15.5.-16.5. Stuttgart

22.5.-23.5. Karlsruhe

- 29.5.-30.5. München

Nach einer Pressemitteilung der

CP GABA GmbH, Hamburg 